\title{
Low temperature light yield measurements in NaI and NaI(TI)
}

\author{
C. Sailer ${ }^{1, \text { a }}$, B. Lubsandorzhiev ${ }^{2}$, C. Strandhagen ${ }^{1}$, J. Jochum ${ }^{1}$ \\ ${ }^{1}$ Kepler Center for Astro and Particle Physics, Auf der Morgenstelle 14, 72076 Tübingen, Germany \\ ${ }^{2}$ Institute for Nuclear Research, Russian Academy of Sciences, pr. Shestidesyatiletiya Oktyabrya 7a, Moscow, Russia
}

Received: 5 March 2012 / Revised: 11 June 2012 / Published online: 27 June 2012

(C) The Author(s) 2012. This article is published with open access at Springerlink.com

\begin{abstract}
The scintillation light output of a pure and a Thallium doped Sodium Iodide (NaI) crystal under irradiation with $5.486 \mathrm{MeV} \alpha$ particles has been measured over a temperature range from $1.7 \mathrm{~K}$ to $300 \mathrm{~K}$. Estimates of the decay time constant at three selected temperatures are given. For pure $\mathrm{NaI}$ an increase in light yield towards low temperatures could be confirmed and measured at higher precision. For $\mathrm{NaI}(\mathrm{Tl})$ below $60 \mathrm{~K}$ an increase in light output has been found.
\end{abstract}

\section{Introduction}

In recent years scintillating crystals at low temperatures used as bolometers have gained interest due to their application in rare event searches, such as the CRESST Dark Matter Search [1] and others, where particle identification is required. Employing different target materials is of special interest in direct Dark Matter Experiments. It allows one to scan the anticipated atomic number $\mathrm{A}^{2}$ dependence of the WIMP-nucleon cross section, that should be present if the scattering is a coherent process. Different materials are investigated at the moment. Here we report on $\mathrm{NaI}$.

As van Sciver and Bogart [2] and Collinson and West [3] reported, the light yield of pure $\mathrm{NaI}$ increases towards lower temperatures. As low temperature bolometers are operated well below liquid helium temperature (e.g. at $\mathrm{mK}$ range) the effort of this work was to extend available data down to $1.7 \mathrm{~K}$ and verify the results reported in [2] and [3]. As a reference a Thallium doped NaI sample of the same geometry was also investigated.

\section{Experimental setup}

The two crystal samples used in this work were produced by Saint-Gobain Crystals. Both samples are cubic shaped

a e-mail: sailer@pit.physik.uni-tuebingen.de with an edge length of $1 \mathrm{~cm}$ where one crystal is doped with Thallium and the other one is made of pure NaI. To ensure that the pure NaI sample was not accidentally doped with Thallium remnants, it was grown in a dedicated mold.

As $\mathrm{NaI}$ is highly hygroscopic the crystals were placed in a copper housing with quartz glass windows manufactured by Aachener Quarz-Glas Technologie Heinrich. The copper holder was assembled in a nitrogen purged glove box and sealed with indium wire. To prevent the remaining nitrogen inside of the capsule from condensing on the optical windows, a custom made cryogenic pump utilizing charcoal was added. An ${ }^{241} \mathrm{Am}$ source with an activity of $840 \mathrm{kBq}$ was placed inside the housing to irradiate the crystal. Since the samples are too small to allow for decent photo absorption rates, a gamma source was not used.

A Janis Research cryostat, model SVT-400, was used to cool down the crystals. The cryostat is equipped with indium-sealed Suprasil II windows transmitting more than $90 \%$ of the light for wavelengths greater than $190 \mathrm{~nm}$. This setup allows using photomultiplier tubes (PMTs) at room temperature, while the sample temperature can be sweeped. The sample is cooled by direct contact with vaporized liquid helium providing temperatures as low as $1.7 \mathrm{~K}$. In order to measure and stabilize the temperature, the cryostat is equipped with two DT-670B-SD diodes by Lakeshore, one measuring the bath temperature at the vaporizer, the other one mounted on the sample holder. A $25 \Omega$ heater on the sample holder is connected to a Lakeshore 331S Temperature Controller, allowing to stabilize temperatures to a precision of up to $0.01 \mathrm{~K}$ or better, depending on the temperature range.

As the maximum of the spectral emission of pure NaI shifts to $300 \mathrm{~nm}$ at lower temperatures [3], type 9235 QB quartz glass tubes produced by ET Enterprises were used. These PMTs provide a high quantum efficiency $\geq 25 \%$ for $\lambda$ between $180 \mathrm{~nm}$ and $440 \mathrm{~nm}$.

The PMT signals were fed into Ortec TF 474 amplifiers where they were amplified and integrated for $20 \mathrm{~ns}$. The sig- 
nal was duplicated in a LeCroy 428F Fan In-Fan Out module ( $\mathrm{FiFo}$ ) and connected to a CAEN N840 leading edge discriminator with low threshold of a few photoelectrons that was used to get a trigger signal. The respective NIM logical pulses were then fed into a LeCroy 622 Quad Coincidence module to realize a logical AND trigger for coincident signals in the two PMTs. The coincident readout is not necessary in terms of background reduction since the signals are sufficiantly high. Yet it allows to reduce the systematical errors. The amplified signal from the FiFo and the trigger pulse were connected to a SIS 3301 FADC that recorded the traces of the scintillation events with a sampling rate of $100 \mathrm{MS} / \mathrm{s}$. For every triggered event the software also recorded both temperature readings. Figure 1 shows a sketch of the setup.

\section{Data analysis}

At each temperature 512,000 scintillation events were recorded from the $\mathrm{NaI}(\mathrm{Tl})$ and 1,024,000 from the pure NaI. A cut was applied to remove traces with nonzero pretrigger mean values. This removes roughly $7 \%$ of the total events. The light output is proportional to the integrated PMT signals. Figure 2 shows an exemplary spectrum in pure $\mathrm{NaI}$ observed by PMT 1 and PMT 2 at a temperature of $178 \mathrm{~K}$. At higher values the peak exhibits a shoulder which can be attributed to double $\alpha$ hits during one record length. These events were not removed by a cut. Rather a fit with three Gaussians was applied to account for baseline noise and both $\alpha$ distributions. The positions of the maximum of the single $\alpha$ distribution were determined for both channels separately and the resulting values were added. Statistical errors are omitted, as they are negligible. Systematical errors have

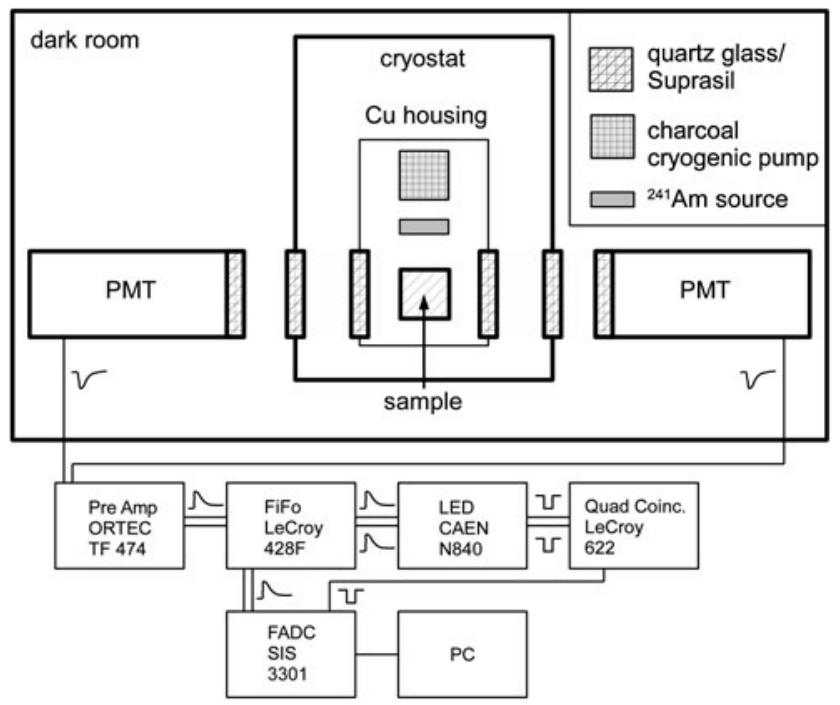

Fig. 1 Sketch of the experimental setup

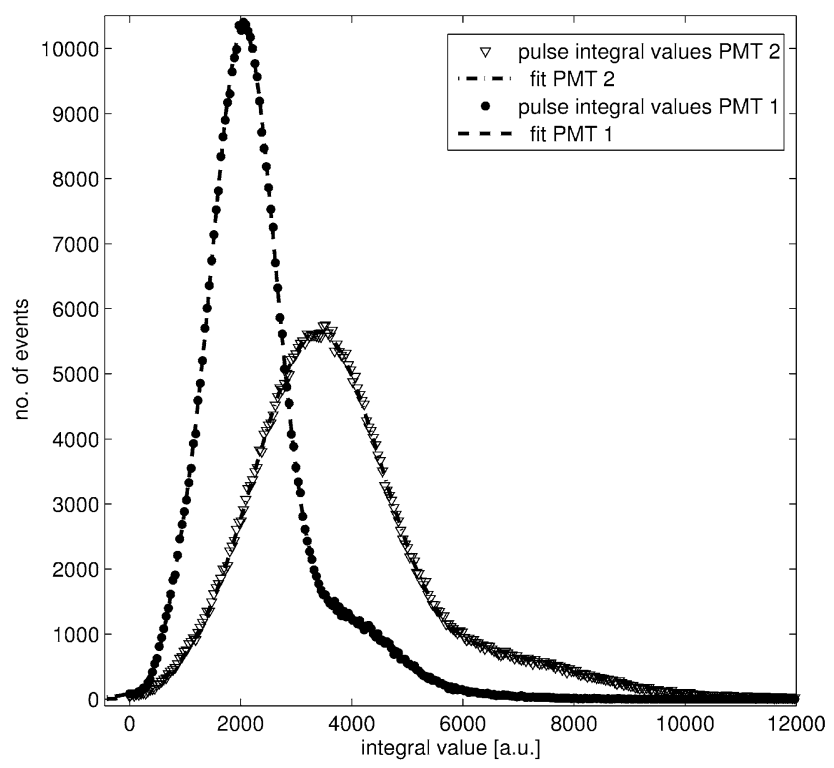

Fig. 2 Exemplary histogram of integral values for scintillation events seen by PMT 1 and PMT 2 at $178 \mathrm{~K}$. The fit accounts for baseline noise and the two $\alpha$ distributions (see text for details)

been derived from variation of the fit, using the $95 \%$ confidence interval for the peak position of the single $\alpha$ peak. The two PMTs were not operated at equal gain due to limitations in the electronics of PMT 2. The gain of PMT 1 was set to a higher value as compared to PMT 2 in order to increase the trigger rate. As all given values are relative quantities, this does not affect the results.

The decay time constants of the signals from the two samples is different, which could already be noticed on the oscilloscope. The pure NaI was recorded with $2.56 \mu$ s record length, the $\mathrm{NaI}(\mathrm{Tl})$ data with $5.12 \mu \mathrm{s}$. To illustrate the temperature dependence, a mean decay time constant was derived in three representative temperature regimes for each crystal. This was done by selecting a sample of scintillation events with a cut on the ratio between pulse height and integral to prevents double events from faking long decay time constants. For each sample a summed pulse was then fitted with two exponential decay times. The $\mathrm{NaI}(\mathrm{Tl})$ has been studied in more details for reasons given in Sect. 4. Note that due to the time resolution of the FADC used, fast components in the order of several nanoseconds can not be resolved. Also the $20 \mathrm{~ns}$ shaping time in the preamplifier will slightly affect the values for $\tau$.

\section{Results}

Figure 3 shows the results for the light yield of pure NaI, Fig. 4 the data for $\mathrm{NaI}(\mathrm{Tl})$ respectively. The light yield axes are scaled such that the room temperature value of $\mathrm{NaI}(\mathrm{Tl})$ is set to one. 


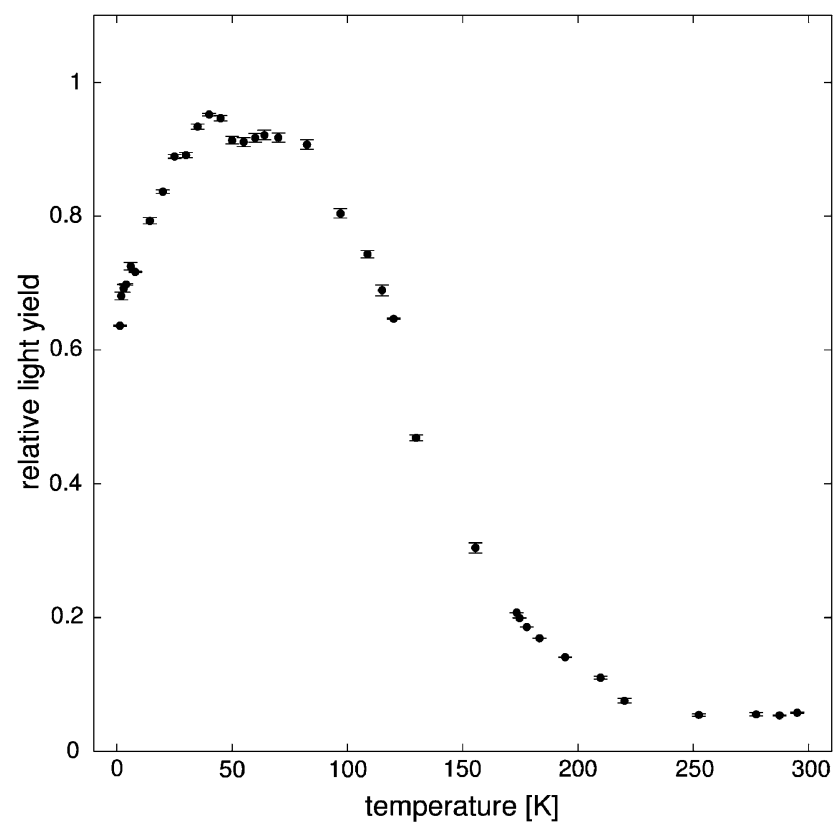

Fig. 3 Light yield of pure $\mathrm{NaI}$ as function of temperature relative to the value of $\mathrm{NaI}(\mathrm{Tl})$ at room temperature. Errors are dominated by systematics, see text

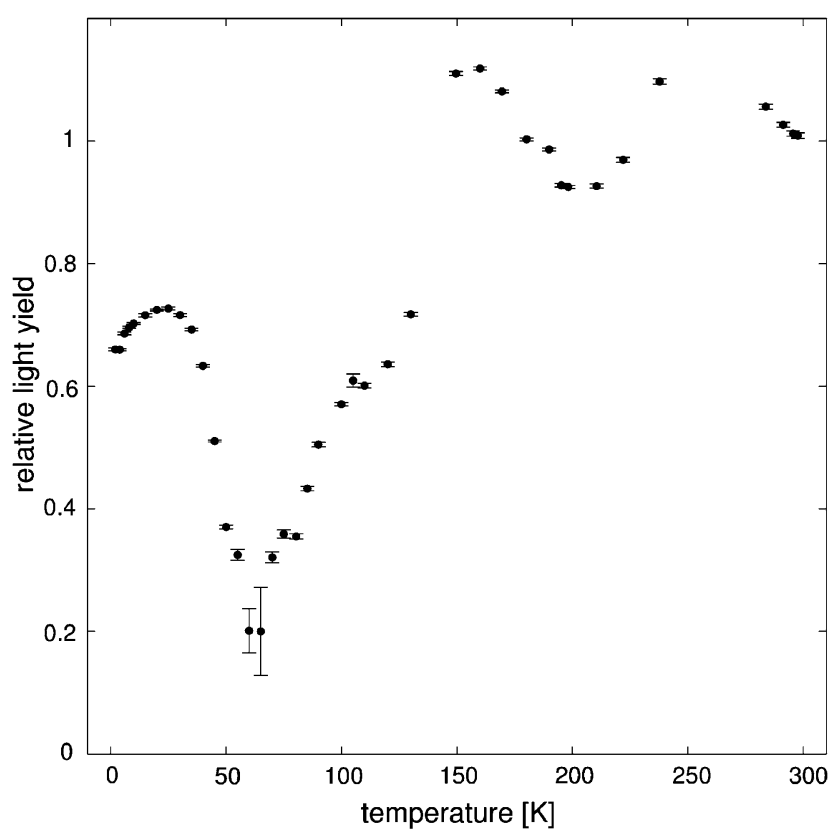

Fig. 4 Light yield of $\mathrm{NaI}(\mathrm{Tl})$ as function of temperature relative to the value at room temperature. Errors are dominated by systematics, see text for details

For pure NaI the general light yield curve published by van Sciver and Bogart [2] is confirmed by our measurement. In this work, data has been slightly extended towards lower temperatures to examine the decrease in light output after the maximum at about $50 \mathrm{~K}$. The temperature resolution has also been increased. It is striking that the maximum value of pure $\mathrm{NaI}$ at around $50 \mathrm{~K}$ is almost comparable to the light yield of Thallium doped $\mathrm{NaI}$ at room temperature. Van Sciver and Bogart reported approximately double the room temperature value of $\mathrm{NaI}(\mathrm{Tl})$, peaking at nitrogen temperature. As there has been quite some progress in producing higher light output $\mathrm{NaI}(\mathrm{Tl})$ crystals since the 1960s the used reference crystal in this work might exhibit a higher light yield, thus giving lower relative values. Also note that in [2] the pulse height was used to deduce the light yield as compared to the integral in this work to take into account the varying decay time. Thus, the maximum of the light emission is found at higher temperatures as compared to Fig. 3 . At $1.7 \mathrm{~K}$ the light yield of pure $\mathrm{NaI}$ amounts to approximately $65 \%$ of the room temperature value of $\mathrm{NaI}(\mathrm{Tl})$.

For $\mathrm{NaI}(\mathrm{Tl})$ a strong suppression in light output is observed down to $60 \mathrm{~K}$. This has been known for quite some time [7]. However we observe a substantial increase in light yield when lowering the temperature further. At $2.0 \mathrm{~K}$ we obtain a value of roughly $65 \%$ relative to the room temperature value of $\mathrm{NaI}(\mathrm{Tl})$ which is comparable to the results obtained for pure NaI.

This raises the question if the increase of light output below $60 \mathrm{~K}$ for $\mathrm{NaI}(\mathrm{Tl})$ could be related to the intrinsic properties of pure $\mathrm{NaI}$ or it is due to another scintillation mechanism. To check this, the decay times at three temperatures have been derived as described in Sect. 3. Results can be found in Table 1.

For pure NaI the decay time slightly increases towards lower temperatures. Van Sciver and Bogart reported the same tendency but give much smaller values, especially at room temperature [2]. Yet Pooley and Runciman [5] state that those decay time values might not be reliable due to trace impurity concentrations.

For the decay time of $\mathrm{NaI}(\mathrm{Tl})$ at $300 \mathrm{~K}$ we find $215 \mathrm{~ns}$ which is in very good agreement with the literature value [6] of $230 \mathrm{~ns}$ at $293 \mathrm{~K}$ which is why we consider the values in this work reliable. Decreasing the temperature of the $\mathrm{NaI}(\mathrm{Tl})$ sample leads to longer decay times peaking around $150 \mathrm{~K}$. Quite unexpectedly at $6 \mathrm{~K}$ the pulses are faster again, even faster than at room temperature. The measured $122 \mathrm{~ns}$ are close to the value of $110 \mathrm{~ns}$ for intrinsic luminescence in

Table 1 Decay times derived from double exponential fit

\begin{tabular}{llll}
\hline NaI pure & & & \\
\hline$T$ & $290 \mathrm{~K}$ & $156 \mathrm{~K}$ & $6 \mathrm{~K}$ \\
$\tau_{1}$ & $98 \mathrm{~ns}$ & $112 \mathrm{~ns}$ & $149 \mathrm{~ns}$ \\
$\tau_{2}$ & - & $644 \mathrm{~ns}$ & - \\
\hline $\mathrm{NaI}(\mathrm{Tl})$ & & & \\
\hline$T$ & $300 \mathrm{~K}$ & $150 \mathrm{~K}$ & $6 \mathrm{~K}$ \\
$\tau_{1}$ & - & - & $23 \mathrm{~ns}$ \\
$\tau_{2}$ & $216 \mathrm{~ns}$ & $646 \mathrm{~ns}$ & $122 \mathrm{~ns}$ \\
\hline
\end{tabular}




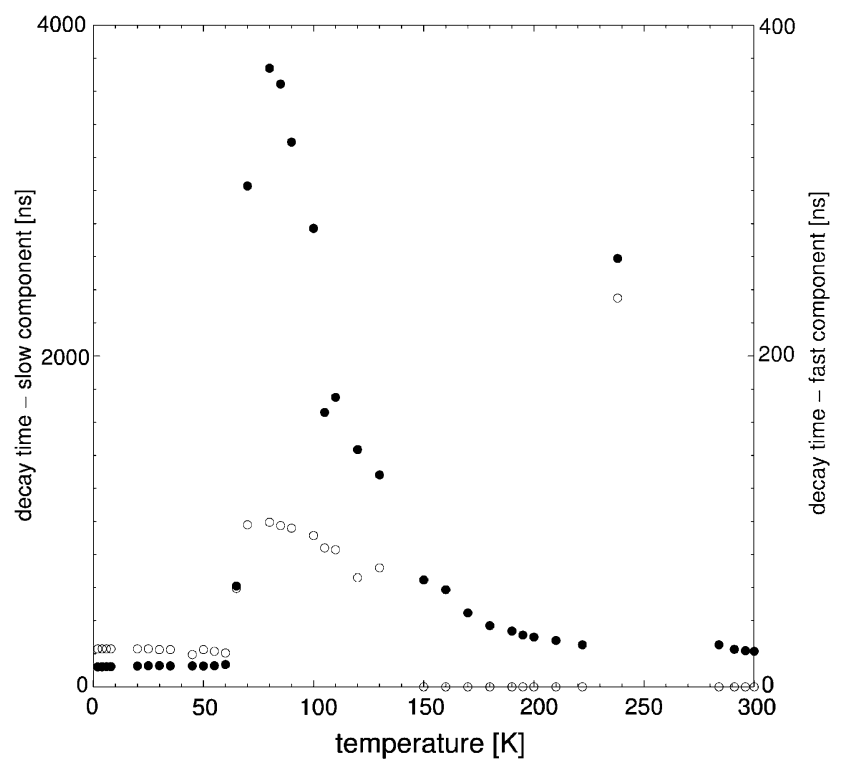

Fig. 5 Decay time behavior for $\mathrm{NaI}(\mathrm{Tl})$. Note the different axes for the slow (solid circles) and fast components (open circles). The sharp drops at around $230 \mathrm{~K}$ and $65 \mathrm{~K}$ correspond to the suppression dips in the light yield curve

pure NaI reported in Fontana et al. [4] for band to band excitation. So it seems possible, that at low temperatures the light yield of $\mathrm{NaI}(\mathrm{Tl})$ is dominated by the intrinsic properties of the NaI lattice. This seems to support the conclusion of Fontana et al. that "at high temperatures the scintillation time constant seems then to be mainly determined by the energy transfer mechanism, and not by the intrinsic behavior of the luminescence center" [4]. To investigate this further, the decay time behavior was studied over the whole temperature range. The result can be found in Fig. 5. The decay times for the fast component are presented using another yaxis scaling as the values are rather small. Two steep drops can be observed at around $230 \mathrm{~K}$ and $65 \mathrm{~K}$, corresponding to the suppression dips in Fig. 4. Below $65 \mathrm{~K}$ the decay times are stable, which is an indication, that no further light yield suppression occurs at lower temperatures. This remains to be verified in a setup at $\mathrm{mK}$ temperatures.

Of course it cannot be excluded that the change in decay time is solely due to the deformation of the unit cell when lowering the temperature. The associated shift in energy, suppressing and delaying the light emission at certain temperatures would also explain the data. The similar light output and decay times at lowest temperature values would then be just considered an uncorrelated coincidence which however seems unlikely.

\section{Conclusion}

In this work we have confirmed that the light yield of pure $\mathrm{NaI}$ increases at low temperatures as reported by van Sciver and Bogart [2]. The data was slightly extended towards lower temperatures and resolved with higher precision than before. At $1.7 \mathrm{~K}$ the light yield of pure $\mathrm{NaI}$ still amounts to about $65 \%$ of the light output of $\mathrm{NaI}(\mathrm{Tl})$ at room temperature.

For $\mathrm{NaI}(\mathrm{Tl})$ an increase in light output has been found below $60 \mathrm{~K}$ which, to our knowledge has not been reported yet and might be attributed to intrinsic luminescence of $\mathrm{NaI}$. At $2 \mathrm{~K}$ for $\mathrm{NaI}(\mathrm{Tl})$ also a value of about $65 \%$ of the room temperature light yield is found.

Acknowledgements The authors would like to thank Claudia Osswald for setting up the cryostat gas handling system. This work was partly funded by SFB-TR 27.

Open Access This article is distributed under the terms of the Creative Commons Attribution License which permits any use, distribution, and reproduction in any medium, provided the original author(s) and the source are credited.

\section{References}

1. G. Angloher et al. (CRESST Collaboration), arXiv:1109.0702v1 (2011)

2. W.J. van Sciver, L. Bogart, IEEE Trans. Nucl. Sci. 5(3), 90-92 (1958)

3. A.J.L. Collinson, J.B. West, J. Phys. B, At. Mol. Phys. 3, 1363 1373 (1970)

4. M.P. Fontana, H. Blume, W.J. van Sciver, Phys. Status Solidi 29, 159-166 (1968)

5. D. Pooley, W.A. Runciman, J. Phys. C, Solid State Phys. 3, 1815$1824(1970)$

6. G.F. Knoll, Radiation Detection and Measurement, 3rd edn. (Wiley, Hoboken, 2000)

7. Bicron, Sodium Iodide-Alkali Halide Scintillation Material (Bicron, Newbury, 1996). Datasheet 